\title{
Crystal Violet Partitioning in Anionic Surfactants Micellar Media: UV-Visible Spectroscopy Study
}

\author{
S. E. Olaseni ${ }^{1 *}$, M. O. Osundiya ${ }^{2}$, E. O. Oniya ${ }^{3}$, O. A. Akeremale ${ }^{1}$, C. O. Aboluwoye ${ }^{1}$, O. E. Oyeneyin ${ }^{1}$ and A. \\ Orunesajo ${ }^{1}$ \\ ${ }^{1}$ Department of Chemical Sciences, Adekunle Ajasin University Akungba, Akoko \\ ${ }^{2}$ Department of Chemistry Lagos State University, Ojo Lagos-Nigeria \\ ${ }^{3}$ Department of Physics and Electronics, Adekunle Ajasin University Akungba, Akoko \\ E-mail: "lincolnolaseni@yahoo.com, segun.olaseni@aaua.edu.ng
}

Received 12 April 2018, Revised 13 August 2018, Accepted 14 August 2018

\begin{abstract}
Simple and differential UV/Visible spectroscopic method were used to monitored the interaction of Sodium dodecyl sulphate (SDS) and Sodium $-N$ - laouryl sarcosinate (SLS) with crystal violet (CV) in pre-micellar and post-micellar concentration range. The results showed that the peak of CV at 590nm was unperturbed in CV/SDS system, but the intensity increased as surfactant concentration increased, unlike in the CV/SLS system. This is an indication that strong interaction between occurred CV and surfactants molecule. Absorption data were also used to obtain partition coefficient $\left(K_{c}\right)$ values and binding constant $\left(K_{\alpha \beta}\right)$ values from which the free energy of partition $\left(\Delta G_{c}^{0}\right)$ and the free energy of binding $\left(\Delta G_{\alpha \beta}^{0}\right)$ were estimated. From the partition coefficient $\left(K_{c}\right)$ values, it could be inferred that the solubilization of $\mathrm{CV}$ is more pronounced and spontaneous in the SDS than in the SLS micellar media.
\end{abstract}

\section{Keywords: Partitioning coefficient; surfactant; sarcosinate; binding constant; micelle.}

\section{Introduction}

Investigations on dye-surfactant interactions in aqueous system have been the line of focus in the last few decades [1-7]. The general picture emerging from these studies is that the peculiar behavior in both absorption spectra and conductivity measurement of dyes [8-10] in the presence of surfactants of opposite charge could be attributed to the formation of a continuum of dye surfactant aggregates [810]. Various techniques, such as spectrophotometry [1114], membrane selective electrode [15], polarography [16], potentiometry [17] and conductometry [18,19] have been used to study the equilibria between surfactant and dye so as to provide information on industrial application like solubilization and on how surfactant assist in fastening of dye in textile industry [20].

Solubilization has been treated as partitioning of additive molecules between a micellar phase and an intermolecular bulk phase [3-5]. Factors like partition coefficient, binding constant, free energies of partition and binding, plus the change in the critical micelle concentration (CMC) of the surfactant in the presence of organic additive, provide useful information relating to the solubilization of additives by micelles [8-10].

Structure of dye and surfactant is important for their mutual interaction as well as dye-fiber and surfactant- fiber interaction, hence, need for optimal surfactant structure for better interaction. Surfactants with different chemical structures give different partition coefficient values for a specific dye. The partition coefficient depends on the structure of the additive, the surfaces that constitute the micelles and other physicochemical variables such as the concentration and temperature.
Crystal violet (CV) is a well-known dye being used for various purposes: a biological stain, a dermatological agent, a veterinary medicine, an additive to poultry feed to inhibit propagation of mold, intestinal parasites and fungus etc. It is also extensively used in textile dying and paper printing [11]. As one of the most widely investigated dyes in terms of its molecular structure, electronic states and the relaxation dynamics of the electronic states [12] with a functional group that is capable of forming hydrogen and covalent bond when interacting with substrate, hence the need for its use as the organic additive in this study.

The surfactants under investigation, for the $\mathrm{CV}$ partitioning, are sodium dodecyl sulfate (SDS) and sodium laouryl $N$-sarcosinate (SLS), which are anionic in nature. As shown in Figure 1, both surfactants have 12-carbon atoms at the hydrophobic end. The main difference in their structure is that, Sodium- $N$ - laouryl sarcosinate has amide bond connecting the hydrophobic tail and the polar head group while sodium lauryl sulfate has a sulfonic acid group instead.

In the present study, the presence of amide and carboxylate groups, at the hydrophilic end of SLS compared with SDS were investigated using spectrophotometric technique to obtain the optimal head group for interaction. The values of partition coefficients $\left(K_{c}\right)$ and equilibrium binding constants $\left(K_{\alpha \beta}\right)$ were compared when they both interact with $\mathrm{CV}$ in aqueous medium. Availability of amide group in SLS, coupled with the carboxylate group is expected to have either negative or positive effect on the interactive power of SLS over SDS. Observation from this study shall provide an insight into the 


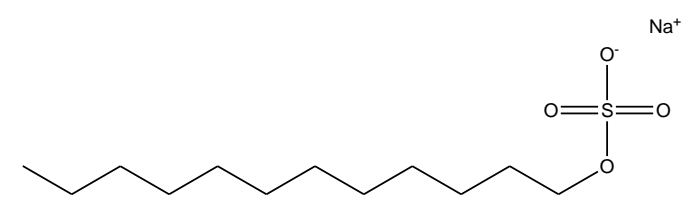

Figurela. Sodium dodecyl sulfate

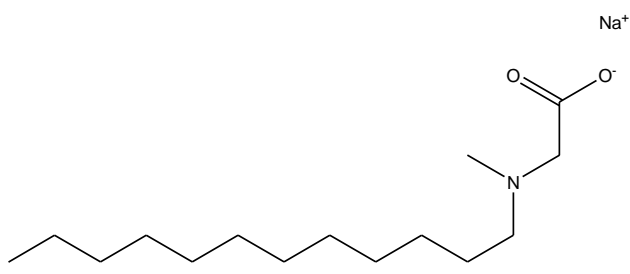

Figure $1 b$. Sodium laouryl $N$-sarcosinate.

Sinopharm Chemical Reagent Co, Ltd. All the experiment solutions were prepared with double distilled water of specific conductance $2-4 \mu_{\mathrm{Scm}^{-1}}$ at $298.15 \mathrm{~K}$. All experiments were carried out at $298.15 \pm 0.2 \mathrm{~K}$, except otherwise stated.

\section{Parameters Calculated}

\subsection{Calculation of Partition and Binding Parameters}

The partition law is the basic law that governs partitioning of additive molecules (dye) between aqueous and micellar medium. The partition coefficient is determined by differential absorbance method reported by Kawamura et al [12]

$$
\frac{1}{\Delta A}=\frac{1}{K_{c} \Delta A_{\infty}\left(C_{a}+C_{s}^{m o}\right)}+\frac{1}{\Delta \mathrm{A}_{\infty}}
$$

$C_{s}$ denotes dye $(\mathrm{CV})$ in $\mathrm{mol} / \mathrm{dm}^{3}, C_{s}{ }^{m o}$ represents the analytical concentration of $\mathrm{CV}$ calculated as $\mathrm{C}_{s^{-}} \mathrm{Cmc}_{0}\left(\mathrm{cmc}_{0}\right.$ is the CMC of the surfactant in water) while $C_{s}$ is the total surfactant concentration. $\Delta A$ represents the differential absorbance while $\Delta \mathrm{A}_{\infty}$ represents its value when $C_{s}$ is at infinity i.e. when $\mathrm{CV}$ is completely solubilized. $K_{c}$ is the partition constant having value in $\mathrm{dm}^{3} / \mathrm{mol}$. The dimensionless partition coefficient $K_{x}$ is related to $K_{c}$ as $K_{x}=$ $K_{c} n_{w}$, where $n_{w}$ is the number of mole of water per $\mathrm{dm}^{3}$ $\left(55.5 \mathrm{~mol} \mathrm{dm}^{-3}\right)$.

The standard free energy change for the transfer of additive from bulk water to micellar phase can be calculated using the relation (28);

$$
\Delta G_{c}^{o}=-2.302 R T \log K_{c}
$$

In Eq. (2), $R$ is the gas constant and $\mathrm{T}$ is the absolute temperature. Binding constant was calculated from

$$
\frac{C_{s} C_{d}}{\Delta \mathrm{A}}=\frac{C_{s}}{\Delta \varepsilon l}+\frac{1}{K_{\alpha \beta} \Delta \varepsilon l}
$$

Here, $C_{d}$ represents the concentration of additive (dye), likewise $C_{s}$, denotes the surfactant concentration. Also, $\Delta \mathrm{A}$ and $\Delta \varepsilon$ represent the differential absorbance and the difference of absorption coefficient, $l$ is path length, while $K_{\alpha \beta}$ stands for the binding constant [13-16]

The standard Gibb free energy of binding can be obtained from the relation;

$$
\Delta G_{\alpha \beta}^{o}=-2.302 R T \log K_{\alpha \beta}
$$

\section{Experimental \\ 3.1 Materials}

Sodium dodecyl sulphate (SDS) and Sodium $N$ - laouryl sarcosinate (SLS) were obtained from Sigma Chemical Company. Analytical grade CV was obtained from

\subsection{Method}

Stock solutions of CV $\left(3.0 \times 10^{-5} \mathrm{moldm}^{-3}\right)$ were prepared by dissolving the dye in distilled water. Stock solution of SDS and SLS $\left(1.0 \times 10^{-2}\right.$ moldm $\left.^{-3}\right)$ surfactants were prepared by dissolving the corresponding amount of each surfactants in distilled water. The absorption spectra of $\mathrm{CV}$ (concentration $=3.0 \times 10^{-5}$ moldm $^{-3}$ ) were recorded in aqueous medium and in micellar media of different surfactants using a double beam UV-2100S spectrophotometer setting, having $0.5 \mathrm{~nm}$ wavelength resolution with $1.0 \mathrm{~cm}$ quartz cell, at $298.15 \mathrm{~K}$. The additive- surfactants interactions, in terms of partition coefficient and binding constant, were quantified from the absorbance spectral data. For simple UV/visible spectra, distilled water was used as the reference, while in differential UV/visible spectroscopy dye solution of a particular concentration was used as the reference.

\section{Results and Discussion \\ 4.1 Interaction of CV with SDS}

The UV-Visible absorption spectrum of $\mathrm{CV}$ in aqueous medium (Figure 2a) displayed maximum absorbance at 590 nm.

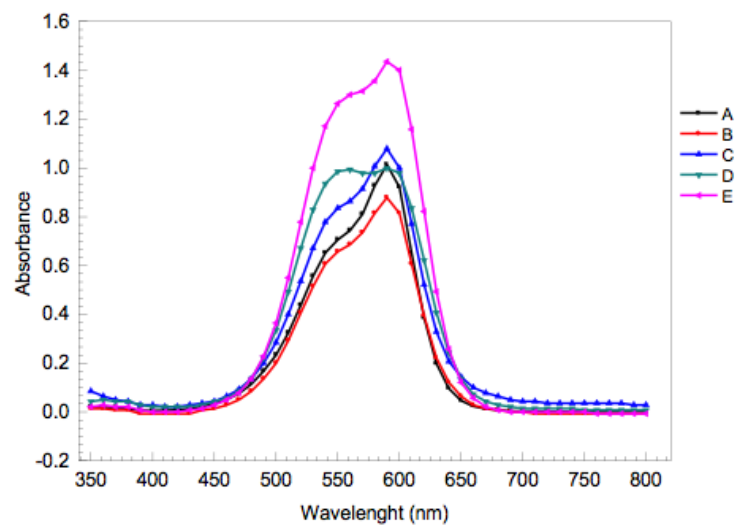

Figure 2a. Absorption spectral of $1.0 \times 10^{-5}$ moldm $^{-3} \mathrm{CV}$ at various concentrations of SDS at $298.15 \mathrm{~K}$. Concentrations of SDS (moldm $\left.{ }^{-3}\right)$ : (A) $2.0 \times 10^{-4}$; (B) $4.0 \times 10^{-4}$; (C) $2.0 \times 10^{-2}$; (D) $4.0 \times 10^{-2}$ and (E) 0.00 .

The absorption spectrum of the dye - surfactant combination (CV/SDS system) was recorded from premicellar to post-micellar region of SDS concentrations $\left(0.002 \mathrm{~mol} / \mathrm{dm}^{3}\right.$ to $\left.0.04 \mathrm{~mol} / \mathrm{dm}^{3}\right)$ and at fixed concentration $\left(1.0 \times 10^{5} \mathrm{~mol} / \mathrm{dm}^{3}\right)$ of $\mathrm{CV}$ in aqueous solution (Figure $2 \mathrm{a}$ ). 
The exhibited maximum absorption at 590mn wavelength in the absence of SDS was unperturbed when SDS was added. The absorption spectral of $\mathrm{CV}$ with different concentrations of the surfactant (i.e SDS) provided insight into the $\mathrm{CV}$-surfactants interactions. The absorbance intensity increased from a value of 0.5812 at $1.0 \times 10^{-4} \mathrm{~mol}$ $\mathrm{dm}^{-3}$ concentration of SDS to a value of 2.104 at $1.0 \times 10^{-2}$ $\mathrm{mol} \mathrm{dm}{ }^{-3}$ concentration of SDS (Figure $2 \mathrm{~b}$ ).

The increasing absorbance intensity ascribed to a manifest of host - guest relationship between CV and SDS. Incorporation of $\mathrm{CV}$ molecules into SDS micelle attracts strong electrostatic interaction between the positive charges on the nitrogen atom of $\mathrm{CV}$ with the ionic groups on the SDS. As shown in Figure $2 b$, the absorbance value increased with increasing concentration of SDS until the critical micelle concentration (CMC) is reached. As soon as the CMC is reached, this absorbance leveled off after reaching a certain value due to maximum incorporation of $\mathrm{CV}$ into the micelles [17-22]. In water, the CMC of SDS was $8.01 \mathrm{mM}$ while in the presence of $\mathrm{CV}$, the value decreased to $1.5 \mathrm{mM}$ due to the modification of coulombic repulsions between the SDS charge head groups and possibility of $\mathrm{CV}$ aggregating to form micelles. These aftermaths made the micellization entropically more favorable and a decrease in the CMC value is expected.

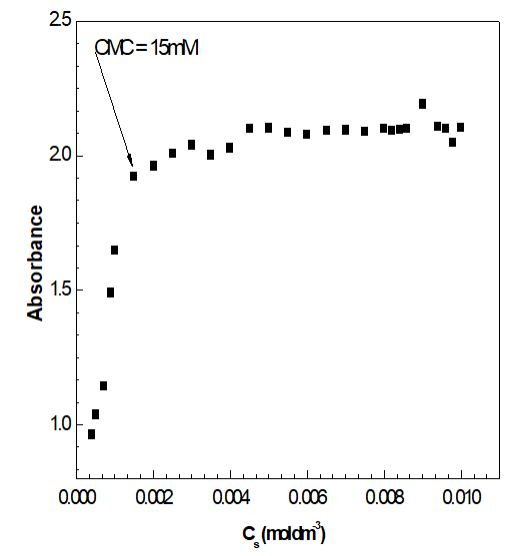

Figure 2b. Plot of simple absorbance of $C V$ as a function of SDS concentration.

The changes in the differential absorbance of CV with varying SDS concentrations are presented in Fig 3a. The values of partition coefficient $\left(K_{c}\right)$ and the binding constant $\left(K_{\alpha \beta}\right)$ for the CV/SDS system were obtained from Fig. 3b and c, respectively.

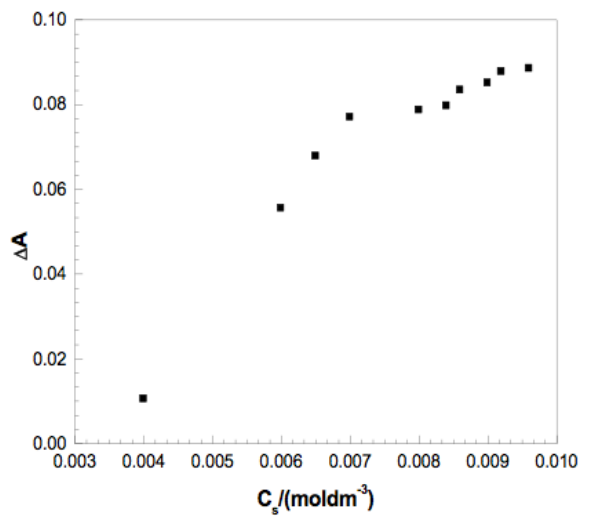

Figure 3a. Plot of differential absorbance of $C V$ as a function of SDS concentrations.
The results showed that the values of partition coefficient $\left(K_{c}\right)$ for the CV/SDS system was $13.32 \mathrm{dm}^{3} \mathrm{~mol}^{-}$ ${ }^{1}$ while the binding constant $K_{\alpha \beta}$ was $8.30 \times 10^{5} \mathrm{dm}^{3} \mathrm{~mol}^{-1}$ (Table 1). Since electrostatic interaction between $\mathrm{CV}$ and anionic SDS molecules (head group) was unhindered, CV molecules were forced to prefer solubilized site in the palisade layer of the micelles hence possible large value of partition coefficient $\left(K_{c}\right)$ and binding constant $\left(K_{\alpha \beta}\right)$ were obtained. The values of $\Delta G_{\alpha \beta}^{o}, \Delta G_{c}^{o}$ and the related equilibrium constant are summarized in Table 1. The negative values of $\Delta G_{c}$ and $\Delta G_{\alpha \beta}\left(-33.2\right.$ and $\left.-8.42 \mathrm{KJmol}^{-1}\right)$ for $\mathrm{CV} / \mathrm{SDS}$ system indicated the spontaneous nature of the partitioning and binding phenomenal as well as the stability of the system [23-29].

Table 1. Partition coefficient $K_{c}$, free energy of partition $\Delta G_{c}$, binding constant $K_{\alpha \beta}$ and free energy of binding $\Delta G_{\alpha \beta}$ for CV/SDS and CV/SLS systems.

\begin{tabular}{lcccc}
\hline Surfactant & $K_{\alpha \beta}$ & $\begin{array}{c}\Delta G_{\alpha \beta} \\
(\mathrm{kJ} / \mathrm{mol})\end{array}$ & $K_{c}$ & $\begin{array}{c}\Delta G_{c} \\
(\mathrm{~kJ} / \mathrm{mol})\end{array}$ \\
\hline SDS & $8.30 \times 10^{5}$ & -8.42 & 13.32 & -33.2 \\
SLS & $1.96 \times 10^{3}$ & -6.03 & 2.86 & -18.8 \\
\hline
\end{tabular}

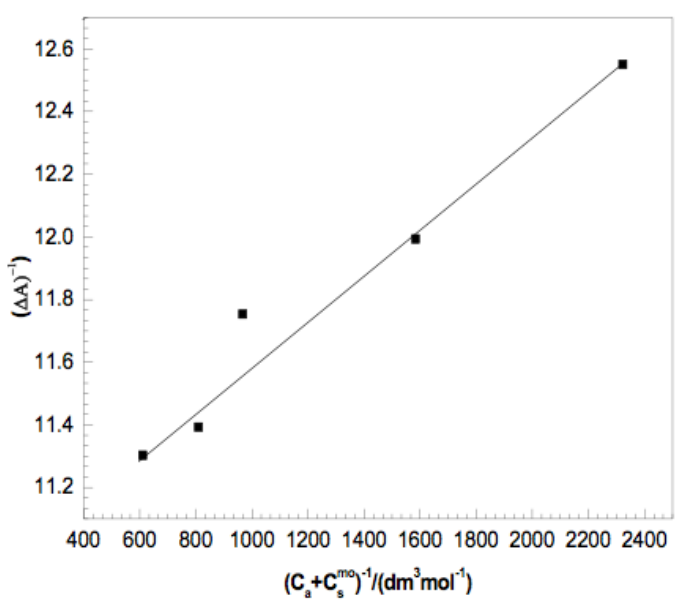

Figure 3b. Relationship between $(\Delta A)^{-1}$ and $\left(C_{s}+C_{s}^{m o}\right)^{-1}$ for the calculation of partition coefficient $\left(K_{c}\right)$ for CV/SDS system.

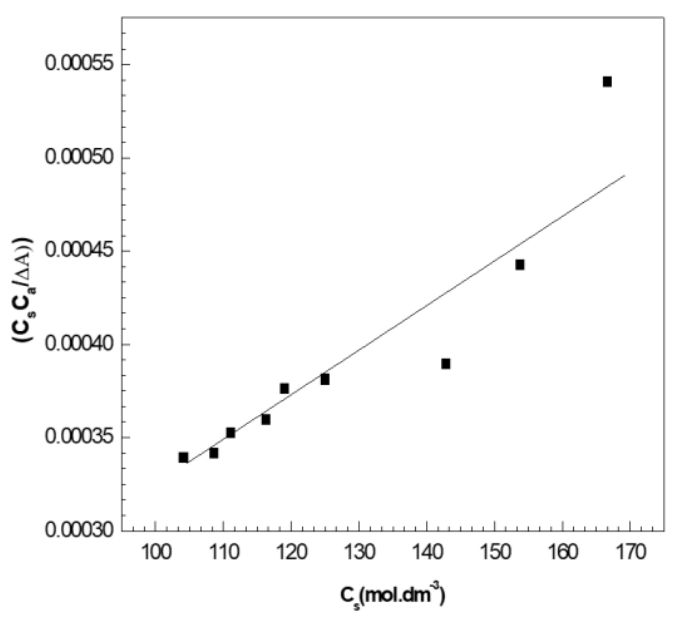

Figure 3c. Plot for calculation of binding constant ( $K_{\alpha \beta}$ ) for CV/SDS system. 


\subsection{Interaction of CV with SLS}

The UV/visible spectrum of CV in the presence of SLS is shown in Figure 4a. It was observed that a red shift in the peak at $590 \mathrm{~nm}$ to $553 \mathrm{~nm}$ occurred. The peak at $553 \mathrm{~nm}$ appeared as a result of the formation of the dimer of the type of the dye which can better be explained in term of dye - surfactant interaction [30].

As the SLS concentrations increase, the dimer disappeared and the peak finally appeared at $596 \mathrm{~nm}$. This indicated strong electrostatic interaction between oppositely charged molecule of SLS [31] and compartmentalization of all $\mathrm{CV}$ molecules into normal micelles [32]. The result presented in Figure $4 \mathrm{~b}$ depicted increase in the absorbance of $\mathrm{CV}$ as the concentration of SLS increased.

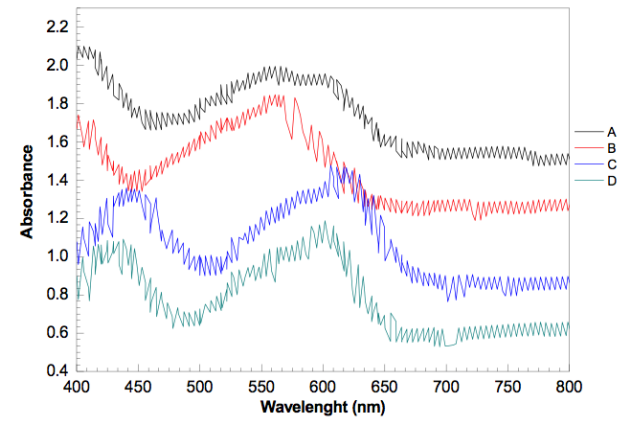

Figure 4a. Absorption spectral of $1.0 \times 10^{-5} \mathrm{moldm}^{-3} \mathrm{CV}$ at various concentrations of SLS at 298.15K. Concentrations of SLS (moldm-3): (A) $2.0 \times 10^{-4}$; (B) $4.0 \times 10^{-4}$; (C) $2.0 \mathrm{X}$ $10^{-2} ; 4.0 \times 10^{-2}$.

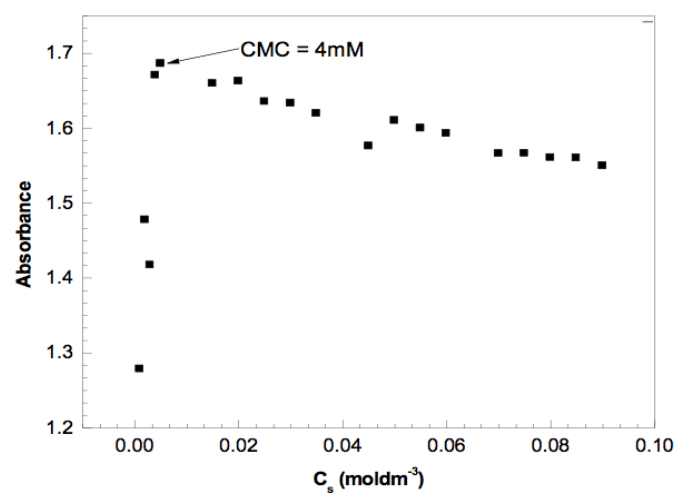

Figure 4b. Plot of absorbance value of $C V$ as a function of SLS concentration.

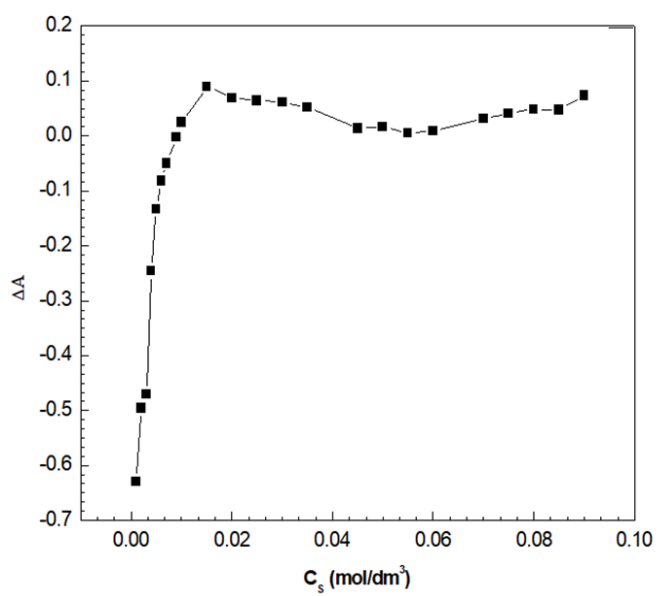

Figure 4c. Plot of differential absorbance of $C V$ as a function of SLS concentrations.

139 / Vol. 21 (No. 3)
This indicated that incorporation of $\mathrm{CV}$ molecules into the micellar core of SLS occurred. Figure $4 c$ showed variation in the differential absorbance of $\mathrm{CV}$ with increasing SLS concentrations.

Partition coefficient $\left(K_{c}\right)$ and binding constant $\left(K_{\alpha \beta}\right)$ values were obtained from Figures $5 \mathrm{a}$ and $5 \mathrm{~b}$, respectively.

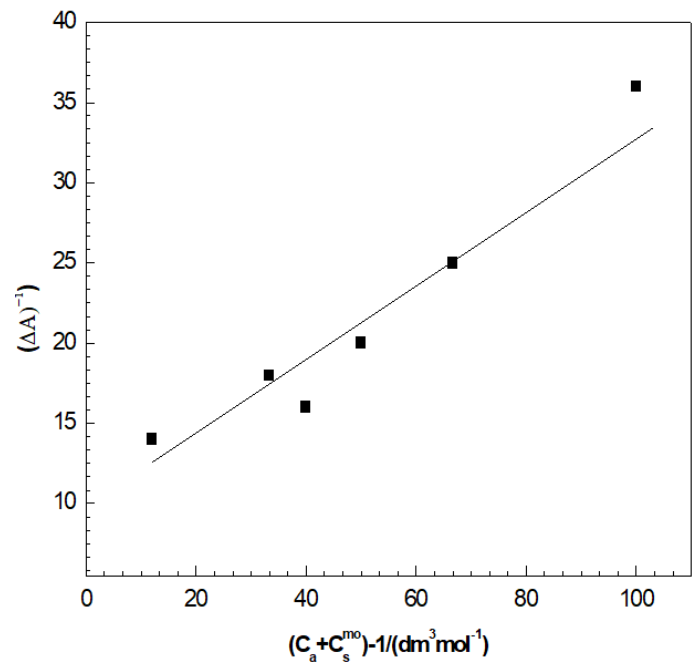

Figure 5a. Relationship between $(\triangle A)^{-1}$ and $\left(C_{s}+C_{s}^{\text {mo }}\right)^{-1}$ for the calculation of partition coefficient $\left(K_{c}\right)$ for $C V / S L S$ system.

The value of the partition coefficient $\left(K_{c}\right)$ was 2.86 , while the value of binding constant $\left(K_{\alpha \beta}\right)$ was $1.96 \times 10^{3}$ (Table 1). Since the amide nitrogen in SLS is methylated, thus, the amide bond cannot be a hydrogen bond donor ( i.e. no formation of hydrogen bonding) which inhibit intermolecular attraction between the amphiphiles aligned at the palisade region and CV molecules. In addition, the steric hindrance of $\mathrm{N}$ - methyl group and the possibility of conformation, cis and trans of the amide bond [33] of SLS may render close alignment of the amphiphiles difficult. All this behavior ascribed to amide bond, $N$-methylated or not, increased the solubility of SLS in water, hence less values of partition coefficient $\left(K_{c}\right)$ and binding constant $\left(K_{\alpha \beta}\right)$ as compared with the CV/SDS system. As a resemblance of CV/SDS system, negative values of $\Delta G_{c}$ and $\Delta G_{\alpha \beta}$ is an indication of spontaneous nature of both partitioning and binding process in this study [23-29].

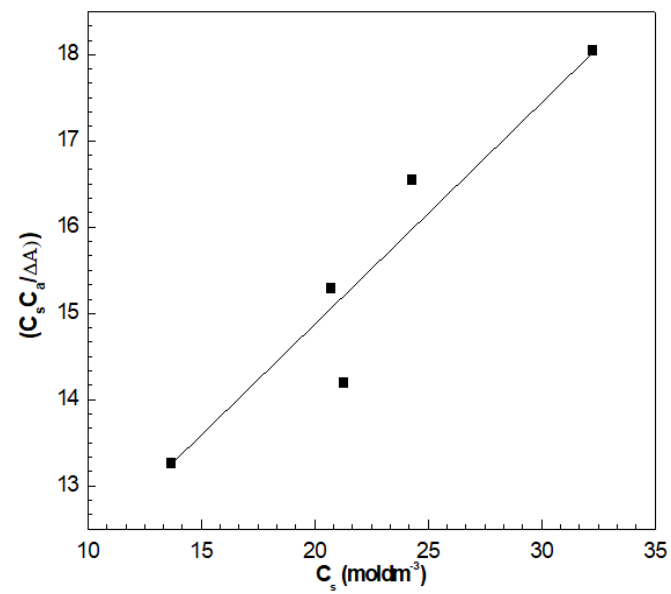

Figure 5b. Plot for calculation of binding constant $\left(K_{\alpha \beta}\right)$ for CV/SDS system.

Int. Centre for Applied Thermodynamics (ICAT) 


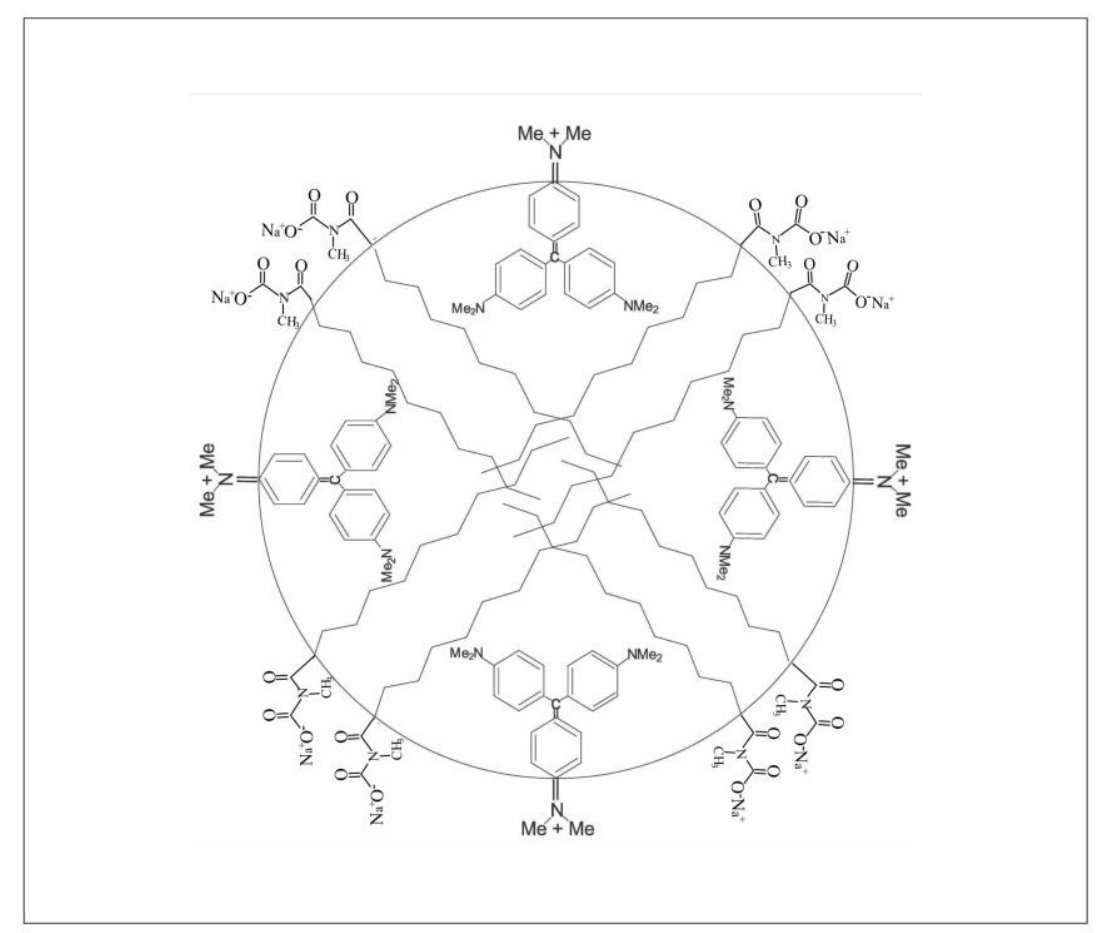

Figure 6a. Proposed locus of CV in SDS micelle.

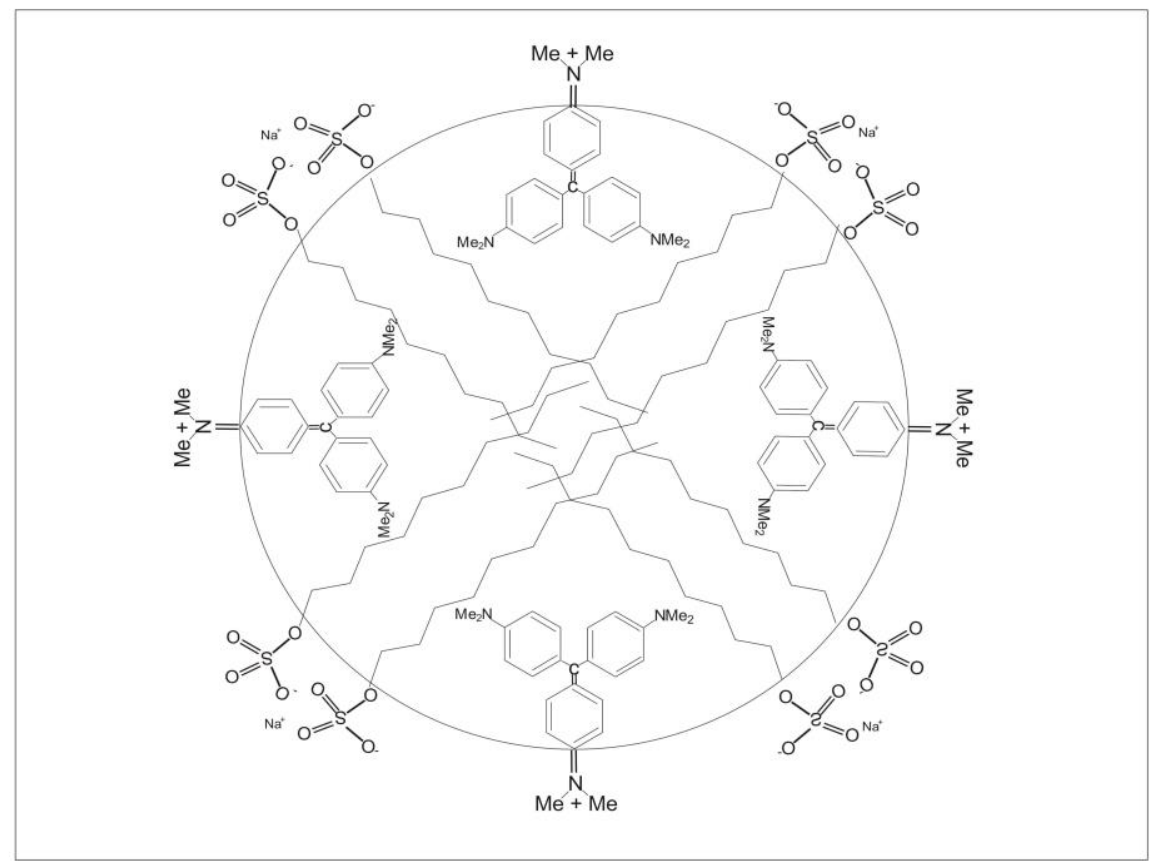

Figure 6b. Proposed locus of CV in SLS micelle.

4.3 Comparison of Interaction of CV with SDS and SLS

SDS has a single functional group while the SLS has double functional groups. Experimental evidence showed that the interaction between the localized positive charge on the $\mathrm{N}$ - atom on the CV and anionic surfactants (SDS and SLS) enhanced electrostatic attraction between the guest and the host.

Indication from Table 1 showed that partition coefficient for both $\mathrm{CV} / \mathrm{SDS}$ and CV/SLS system were greater than one. This is an indication that $\mathrm{CV}$ is more soluble in the micelle medium than in aqueous medium. This observation is more pronounced in CV/SDS system. This was ascribed to the fact that the electrostatic interaction between $\mathrm{CV}$ and SDS were unhindered at the palisade layer, where the SDS molecules are anchored with their head groups at the interface toward water. Figure 6a shows proposed locus of $\mathrm{CV}$ in SDS micelle. In the case of CV/SLS system, there is difficulty in alignment of the amphiphiles because of the $N$ methylated amide bond, hence some CV molecule were forced to be solubilized in the aqueous system which reduced the quantity of $\mathrm{CV}$ solubilizing at the palisade layer, resulting in reduction in the partition coefficient value. Figure $6 \mathrm{~b}$ shows proposed locus of $\mathrm{CV}$ in SLS micelle. Higher binding constant values were obtained in the CV/SDS system relative to the CV/SLS system, where the value was small. This observation was ascribed to the negative inductive effect of the alkyl chain of both surfactants, leading to a decrease in electron density on - 
$\mathrm{SO}_{4}^{-}$group in the SDS than it can do on $-\mathrm{COO}^{-}$in the case of SLS. This action on CV/SDS system in palisade layer enhanced strong electrostatic interaction between $\mathrm{CV}$ and SDS molecules. Hence, a high value of binding constant was obtained for the CV/SDS system (Table 1)

\section{Conclusions}

In this study, the determination of the level of interaction between $\mathrm{CV}$ and anionic surfactants (SDS and SLS) as indicated by absorption spectrum, showed that there was interaction between the dye and the surfactant molecule. Despite the fact that SDS and SLS have the same hydrophobic tail, it was revealed that the charge head group of the surfactant molecule played a prominent role in the dye - surfactant interaction and dye solubilization. Results from this study showed that under the same experimental condition, CV/SDS system has much higher partition coefficient and binding constant values relative to the CV/SLS system. The interpretation of this is that, the degree of solubilization of CV/SDS system was greater than that of CV/SLS system. Partitioning was spontaneous in both systems but it is well pronounced in the case of the SDS micelles.

\section{Acknowledgements}

Authors wish to thank Tertiary Education Trust Fund (TETFund), Nigeria, for the financial support.

\section{References}

[1] F. Gul, A.M. Khan, S.S. Shah, M.F. Nazar, "Spectroscopic study of alizarin reds binding with cetythimethylammonium bromide at low concentrations", Color. Technol., 126, 109-113, 2010. DOI: $10.1111 /$ j.1478-4408.2010.00236.x

[2] S.S. Shah, G.M. Laghari, H. Kashif, S.W.H. Shah, "Partition coefficient of amphilphilic hemicyanine dyes between the aqueous and the micellar phase of sodium dodecylsulfate by different absorbance spectroscopy," Colloids and surfaces A. Physicochemical and Engineering Aspect 143, 111-115, 1998. DOI: 10.1016/S0927-7757(98)00496-8

[3] C.A. Bunton, L. Sepulveda, "Hydrophobic and coulombic interactions in the micellar binding of phenols and phenoxide ions" J. Phys. Chem. 83, 680683, 1979. DOI: $10.1021 / \mathrm{j} 100469 \mathrm{a} 008$

[4] A. S. George, D.C. Sherril, E.T. Edwin, F.S. John, "Solubilization of hydrocarbons by surfactant micelles and mixed micelles," J. Colloid Inter. Sci. 130, 254265.1989. DOI: 10.1016/0021-9797(89)90097-0

[5] R. S. Tsai, W. Fan, N.El. Tayer, P.A. Carrupt, B. Testa, L.B. Kier, "Solute-water interactions in the organic phase of a biphasic system. 1. Structural influence of organic solutes on the "water-dragging" effect" J. Am. Chem. Soc. 115, 9632-9639, 1993. DOI: $10.1021 / \mathrm{ja} 00074 \mathrm{a} 031$

[6] M.A. Cheema, P. Taboada, S. Barbosa, M. Siddiq, V. Mosquera, "Effect of molecular structure on the hydration of structurally related antidepressant drugs," Mol. Phy. 104, 3203-3212.2006, DOI: $10.1080 / 00268970601070460$
[7] Y. Rangel, A. Jr. Pessoa, L.C. Tavares, "Micellar solubilization of drugs," J. Pharm. Pharm. 8, 142-163 2005. PMID 16124926

[8] D. Attwood, A.T. Florence, (1983) "Surfactant Systems." Springer, Netherlands

[9] A. M. Khan, S.S. Shah, "Determination of critical micelle concentration $(\mathrm{cmc})$ of sodium dodecyl sulphate (SDS) and effect of low concentration of pyrene on its cmc by using ORIGIN software." J. Chem. Soc. Pak. 30, 186-191 2008.

[10] P. Taboada, J.M. Ruso, M. Garcia, V. Mosquera, "Surface properties of some amphiphilic antidepressant drugs." Colloids Surf. A 179, 125-128 2001. DOI: 10.1016/S0927-7757(00)00730-5

[11] M. Sarkar, S. Poddar, "Studies on the Interaction of Surfactants with Cationic Dye by Absorption Spectroscopy" J. Colloid Interface Sci. 221, 181-185, 2002. DOI: $10.1006 /$ jcis. 1999.6573

[12] S.A. Moore, K.M. Glenn, R.M. Palepu, "Spectroscopic Investigations on the Interaction of Crystal Violet with Nonionic Micelles of Brij and Igepal Surfactants in Aqueous Media." J. Solution Chem. 36, 563-571, 2007. DOI: $10.1007 / \mathrm{s} 10953-007-9133-6$

[13] M.F. Nazar, M. Abid, M. Danish, M. Ashfaq, A.M. Khan, M.N. Zafar, S. Mehmood, A. Asif, "Impact of L-leucine on controlled release of ciprofloxacin through micellar catalyzed channels in aqueous medium." $J$. Mol. Liq. 212, 142-150, 2015. DOI: 10.1016/j.molliq.2015.09.015

[14] M.F. Nazar, F. Mukhtar, S. Chaudry, M. Ashfaq, S. Mehmood, A. Asif, U.A. Rana, "Biophysical probing of antibacterial gemi-floxacin assimilated in surfactant mediated molecular assemblies." J. Mo.l Liq. 200, 361368, 2014. DOI: 10.1016/j.molliq.2014.11.007

[15] O. Duman, S. Tunc, B.B. Kanci, "Spectrophotometric studies on the interactions of C.I. Basic Red 9 and C.I. Acid Blue 25 with hexadecyltrimethylammonium bromide in cationic surfactant micelles." Fluid Phase Equilibria 301, 56-61, 2011. DOI: 10.1016/j.fluid.2010.11.018

[16] M.F. Nazar, F. Mukhtar, M. Ashfaq, H.M.A.U. Rahman, M.N. Zafar, S.H. Sumrra, "Physicochemical investigation of antibacterial moxifloxacin interacting with quaternary ammonium disinfectants." Fluid Phase Equilibria 406, 47-54, 2015. DOI: 10.1016/j.fluid.2015.07.033

[17] M. Usman, A. Khan, M. Siddiq, "Thermodynamic and solution properties of amphiphilic anti-allergic drug cetirizine HCl.” J. Chem. Soc. Pak. 31, 221-227, 2009.

[18] M. Usman, A. Khan, M. Siddiq, "Thermodynamic properties of amphiphilic anti-depressant drug citalopram HBr.” J. Chem. Soc. Pak. 32, 1-6, 2010.

[19] A. Shah, A.M. Khan, M. Usman, R. Qureshi, M. Siddiq, S.S. Shah, "Thermodynamic characterization of 
dexamethasone sodium phosphate and its complex with DNA as studied by conductometric and spectroscopic techniques.” J. Chilean Chem. Soc. 54, 134-137, 2009.

[20] M. Usman, M. Siddiq, "Surface and micellar properties of chloroquine diphosphate and its interactions with surfactants and human serum albumin." J. Chem. Thermodyn. 58, 359-366, 2013. DOI: 10.1016/j.jct.2012.11.022

[21] M. Usman, M. Siddiq, "Probing the micellar properties of quinacrine $2 \mathrm{HCl}$ and its binding with surfactants and human serum albumin." Spectrochim Acta Part A 113, 182-190, 2013. DOI: 10.1016/j.saa.2013.04.089

[22] M. Usman, M.A. Rashid, A. Mansha, M. Siddiq, "Thermodynamic solution properties of pefloxacin mesylate and its interactions with organized assemblies of anionic surfactant, sodium dodecyl sulphate." Thermochim Acta 573, 18-24, 2013.DOI: 10.1016/j.tca.2013.08.014

[23] M.F. Nazar, S.S. Shah, M.A. Khosa, "Interaction of Azo dyes with cationic surfactant under different $\mathrm{pH}$ conditions." J. Surfact Deterg. 13, 529-537, 2010. DOI: $10.1007 / \mathrm{s} 11743-009-1177-8$

[24] M.J. Schick, F.M. Fowkes, "Foam stabilizing additives for synthetic detergents. interactions of additives and detergents in mixed micelles." J. Phys. Chem. 61, 1062-1068, 1957. DOI: $10.1021 / \mathrm{j} 150554 \mathrm{a} 007$

[25] M. Sarkar, S. Poddar, "Studies on the interaction of surfactants with cationic dye by absorption spectroscopy." J. Coll. Interface Sci. 221, 181-185, 2000. DOI: $10.1006 /$ jcis. 1999.6573

[26] H. Akbas, T. Taner, "Spectroscopic studies of interaction between C.I. Reactive Orange 16 with alkyltrimethylammonium bromide surfactants." Spectrochimica Acta. Part A, Molecular and
Biomolecular Spectroscopy 73, 150-153, 2009. DOI: 10.1016/j.saa.2009.02.018

[27] S.S. Shah, G.M. Laghari, K. Naeem, S.W.H. Shah, "Partition coefficient of amphiphilic hemicyanine dyes between the aqueous and the micellar phase of sodium dodecyl sulfate by differential absorbance spectroscopy." Coll. Surf. A 143, 111-115, 1998. DOI: 10.1016/S0927-7757(98)00496-8

[28] K. Naeem, S.S. Shah, S.W.H. Shah, G.M. Laghari, "Solubilization of cationic hemicyanine dyes in anionic surfactant micelles: a partitioning study." Monatsh Chem. 131, 761-767, 2000. DOI: $10.1007 / \mathrm{s} 007060050023$

[29] S.S. Shah, K. Naeem, S.W.H. Shah, G.M. Laghari, "Differential absorbance measurements of amphiphilic hemicyanine dyes, solubilization study in anionic surfactant." Coll. Surf. A 168, 77-85, 2000. DOI: 10.1016/S0927-7757(99)00520-8

[30] G. Soumen, M. Satyajit, D. Sibani, B. Rathin, "Spectroscopic investigation of interaction between crystal violet and various surfactants (cationic, anionic, nonionic and gemini) in aqueous solution." Fluid Phase Equilibria 332, 2012. DOI: 10.1016/j.fluid.2012.06.019

[31] L. Garc1'a-R1'o, P. Hervella, J.C. Mejuto, M. Parajo, $\mathrm{M}$, "Spectroscopic and kinetic investigation of the interaction between crystal violet and sodium dodecylsulfate" Chemical Physics 335, 164-176, 2007. DOI: 10.1016/j.chemphys.2007.04.006

[32] S. Mitali, P. Swapan, "Studies on the Interaction of Surfactants with Cationic Dye by Absorption Spectroscopy." J. Coll. Interface Sci. 221, 181-185, 2000. doi:10.1006/jcis.1999.6573,

[33] R. Bordes, T. Juergen, H. Krister, "Role of an Amide Bond for self - Assembly of surfactants." Langmuir 26, 3077-3083，2009. DOI: 10.1021/1a902979m 